\section{Measurement system for two-dimensional magnetic field distributions, applied to the investigation of recording head fields}

\section{J P J Groenland and J H J Fluitman}

Twente University of Technology, Department of Electrical Engineering, PO Box 217, 7500 AE Enschede, The Netherlands

\section{Received 8 July 1980 in final form 18 September 1980}

Abstract A measurement system is described that can be used for the analysis of two-dimensional microscopic field structures like those produced by magnetic recording heads. The system is built around a very accurate positioner into which a sensitive transducer and the object of analysis is mounted.

The properties of the applied magnetoresistive transducer are described. This transducer, a very narrow permalloy strip placed at the edge of a glass substratum, can be used to measure both components of the field distribution.

The analysis of the measured results can be accomplished with the help of a computer simulation of the transducer response curves. The performance of the system is demonstrated by measurements on a number of ferrite heads and conclusions about the so called 'dead layer'structures on these heads are given.

\section{Introduction}

The evolution of magnetic recording systems like digital disc and tape memories and video recording systems is charac- terised by a proceeding miniaturisation of the recording process with ever decreasing dimensions of the recording heads and head gap lengths, decreasing head-medium separation and recording layer thickness. Since a detailed knowledge of the magnetic field structure of recording heads and of the magnetised medium is essential for further progress, the trend to miniaturisation is followed by an increasing need for accuracy and resolving power of the analysis equipment.

The measurement technique which is described in this paper can be helpful in analysing the magnetic field structure of recording transducers and media and any other suitable two-dimensional field configuration. Some rough information about the equipment has been published before in the context of the presentation of recording head field measurements (Fluitman 1978, Fluitman and Groenland 1979), but it is the purpose of this paper to disclose the details. We describe the possibility of measuring the two components of a magnetic vector field by means of one and the same magnetoresistive transducer.

In figure 1 the scheme of the system, which essentially is a high precision positioner to bring a transducer and an object to be measured into a well defined mutual position, is presented. The transducer and the positioner are described in the next two sections. In $\$ 4$ and 5 the measurements on ferrite recording heads are presented.

\section{The transducer}

The magnetoresistive transducer (MRT) consists of a vacuum deposited permalloy strip and is placed at the edge of a glass substratum (see figure 2 ). The substratum has the form of a cube with dimensions $20 \times 20 \times 20 \mathrm{~mm}$. The reason for this will be made clear in $\$ 3$. The dimensions of the permalloy strips used until now are typically: length $L=50 \mu \mathrm{m}$; width $w=2.0 \mu \mathrm{m}$; thickness $t=20 \mathrm{~nm}$.

The choice of the MRT as a transducer (and not a Hall generator (Lustig et al 1979), for instance) has been made because of its sensitivity, the relative ease of production on a substratum edge (Fluitman and Krabbe 1972) and the possibility of measuring two field components. Of course an MRT has drawbacks too. They will be described at the end of this section. The magnetoresistance of permalloy strips has been treated at several places in literature (for instance West 1961). For an applied magnetic field $H_{\mathrm{a}}$ in the plane of the strip but perpendicular to its axis the behaviour is described by the

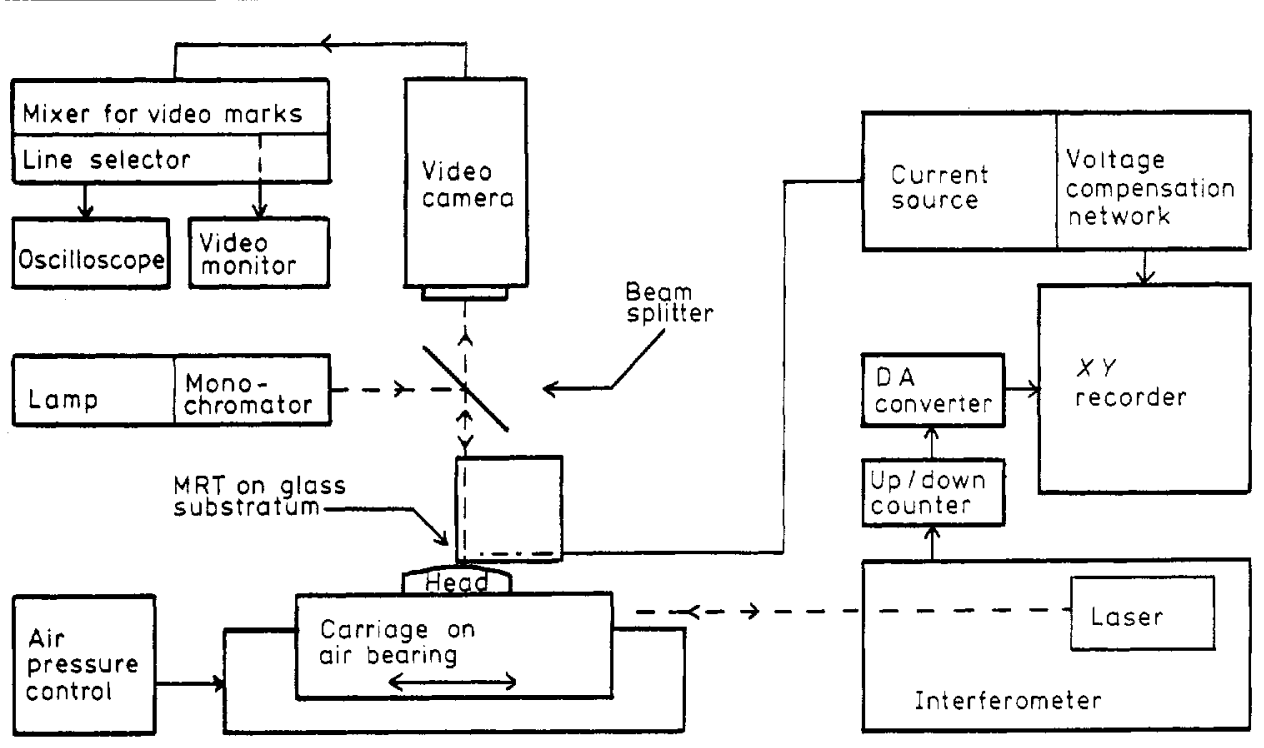

Figure 1 Scheme of the measurement system. 


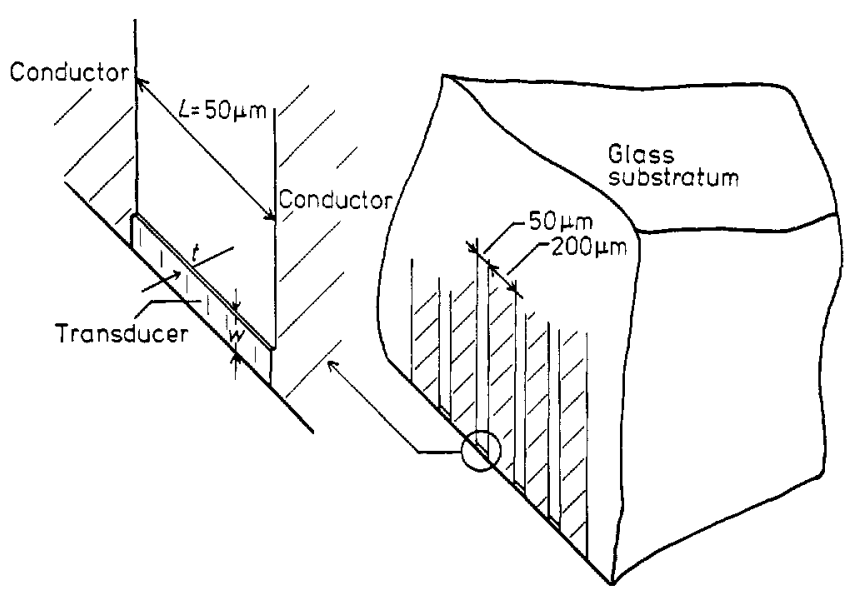

Figure 2 A magnetoresistive transducer, deposited on the edge of a glass substratum.

following set of equations which are locally valid:

$$
\begin{aligned}
\sin \phi & =\frac{M_{y}}{M_{\mathrm{s}}}= \begin{cases}H / H_{\mathrm{k}} & \left(|H| \leq H_{\mathrm{k}}\right) \\
\pm 1 & \left(|H|>H_{\mathrm{k}}\right)\end{cases} \\
H & =H_{\mathrm{a}}+H_{\mathrm{dem}} \\
H_{\mathrm{dem}} & =\frac{1}{\pi} \int_{-w / 2}^{+w / 2} \frac{\mathrm{d} M_{\mathrm{s}} \sin \phi\left(y_{0}\right)}{\mathrm{d} y_{0}} \tan ^{-1} \frac{t}{2\left(y_{0}-y\right)} \mathrm{d} y_{0}
\end{aligned}
$$

with $\phi$ the rotation of the saturation magnetisation $M_{\mathrm{s}}$ with respect to the easy axis. This angle must be considered as a function of $y$ (or the integration variable $y_{0}$ ). $M_{y}$ is the $y$ component of the magnetisation and is equal to $M_{\mathrm{s}} \sin$ $\phi\left(y_{0}\right) . H_{k}$ is the constant of uniaxial anisotropy and $w$ and $t$ the width and thickness of the transducer respectively (see figure 3). There is no need for the field $H_{\mathrm{a}}$ to be homogeneous

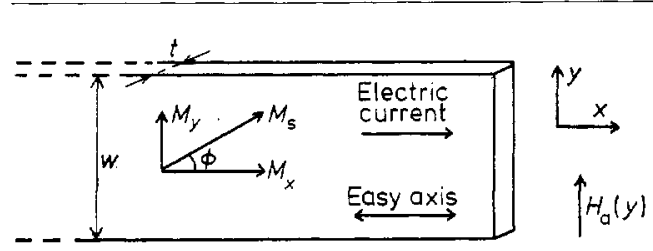

Figure 3 Rotation of the local magnetisation vector $\boldsymbol{M}$ in a permalloy strip by an applied magnetic field in the $y$ direction.

in the $y$ direction, so in general $H_{\mathrm{a}}$ is a function of $y$. The expression for the transducer resistance $(R)$ reads:

$$
R=R_{0}-\frac{\Delta R}{w} \int_{-w / 2}^{w / 2}\left(\frac{M_{y}}{M_{\mathrm{s}}}\right)^{2} \mathrm{~d} y_{0}
$$

with $R_{0}$ the resistance in zero field and $\Delta R$ the maximum magnetoresistance effect.

From these equations it is obvious that as long as $M_{y}<M_{\mathrm{s}}$ everywhere in the strip (no saturation), the equations determining $M_{y}$ are linear with respect to $H_{\mathrm{a}}$ which means that the magnetoresistance is quadratic in the amplitude of $H_{\mathrm{a}}$, whatever the form of $H_{\mathrm{a}}$. If $H_{\mathrm{a}}$ is homogeneous, for instance during calibration of the transducer, and $H_{\mathrm{k}} \gg t M_{\mathrm{s}} / w$ one finds:

$$
\begin{array}{ll}
R=R_{0}-\Delta R\left(H_{\mathrm{a}} / H_{\mathrm{k}}\right)^{2} & \left(H_{\mathrm{a}} \leq H_{\mathrm{k}}\right) \\
R=R_{0}-\Delta R & \left(H_{\mathrm{a}}>H_{\mathrm{k}}\right)
\end{array}
$$

If the condition $H_{\mathrm{k}} \gg t M_{\mathrm{s}} / w$ is not fulfilled one can generally write $R=R_{0}-\alpha \Delta R\left\{H_{\mathrm{a}} /\left[H_{\mathrm{k}}+\left(t M_{\mathrm{S}} / w_{)}\right)\right\}^{2}\right.$ for the case that there is no saturation. Here we have followed the practice of normalising $H_{\mathrm{a}}$ on $\left[H_{\mathrm{k}}+\left(t M_{\mathrm{s}} / w\right)\right]$. The coefficient $\alpha$ is a function of $\left(t M_{\mathrm{s}} / w\right) /\left[H_{\mathrm{k}}+\left(t M_{\mathrm{s}} / w\right)\right]$. We have computed this function which is depicted in figure 4 . In the limiting case when $H_{\mathrm{k}} \ll t M_{\mathrm{s}} / w, \alpha$ goes to $\frac{2}{3}$ provided that $t \ll w$. (It must be noted that $t M_{\mathrm{s}} / w$ loses its meaning as an approximation of demagnetising fields when $t \leqslant w$ is no longer true.) Computer calculations have shown that the upper boundary of validity of the formula $R=R_{0}-\alpha \Delta R\left\{H_{a} /\left[H_{\mathrm{k}}+\left(t M_{\mathrm{s}} / w\right)\right]\right\}^{2}$ is about $H_{\mathrm{a}}=H_{\mathrm{k}}+\left(t M_{\mathrm{s}} / w\right)$ but we have no proof whether or not this is the exact boundary. A general expression for the magnetoresistance in the saturating case is not known to us.

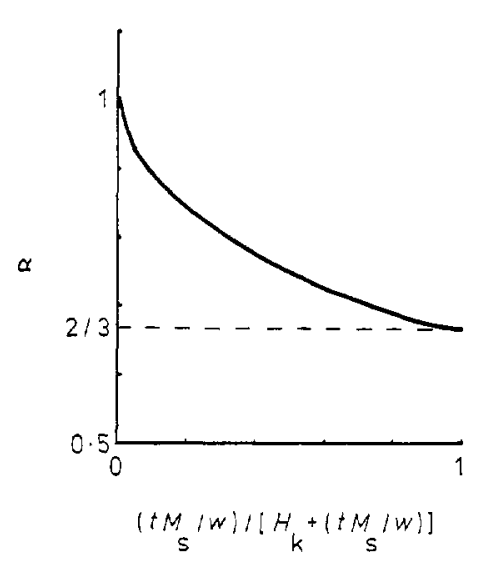

Figure 4 The relation between $\alpha$ and $\left(t M_{\mathrm{s}} / w\right) /\left[H_{\mathrm{k}}+\left(t M_{\mathrm{5}} / w\right)\right]$

Most of the experimental strips we have produced do not fit entirely to the theoretical curves, while some do. The most general departure from theory is that $R$ is slightly too large in the region $H \sim H_{\mathrm{k}}+\left(t M_{\mathrm{s}} / w\right)$ (see figure 5). Transducers are selected for our purposes when the deviation from theoretically predicted behaviour does not exceed a few per cent in the applied range.

From the calibration the magnitude of $H_{\mathrm{k}}$ and $t$ (which are not determined separately) are derived since these parameters are needed for the analysis of measurements on non-homogeneous fields. All transducers we use, have $H_{\mathrm{k}} \ll t M_{\mathrm{s}} / w$, i.e. the magnetic anisotropy is dominated by the geometry of the strip. Practical values of $t M_{\mathrm{s}} / \mathrm{w}$, which define the ranges of the transducers are $3000-15000 \mathrm{~A} / \mathrm{m}$ (while $H_{\mathrm{k}}$ is in the order of $200 \mathrm{~A} / \mathrm{m}$ ).

The sensitivity of the MRT is proportional to the transducer current $i_{\mathrm{t}}$. However, to prevent heating of the transducer (necessary to prevent errors as a consequence of the temperature dependence of $R$ ) this current must be limited. This means that the maximum transducer response $i_{\mathrm{t}} \Delta R$ is in the order of $1 \mathrm{mV}$ in (our) practice. The temperature coefficient of the transducer $\left(\sim 4 \% /{ }^{\circ} \mathrm{C}\right)$ is relatively large in view of the maximum value of the magnetoresistive effect. $\left(\Delta R / R_{0} \simeq 2 \%\right.$. For this reason DC field measurements on objects with temperature gradients may be inaccurate. In such a case one must consider an AC mode of measurement. The MRT is sensitive to magnetic field components which are in the plane of the permalloy strip. Perpendicular components are negligible in all practical cases as a consequence of the large demagnetising factor $(\sim 1)$ in this direction. Because of its shape the transducer is rather sensitive to fields which are parallel to the strip axis. These parallel fields introduce a decreased transducer 


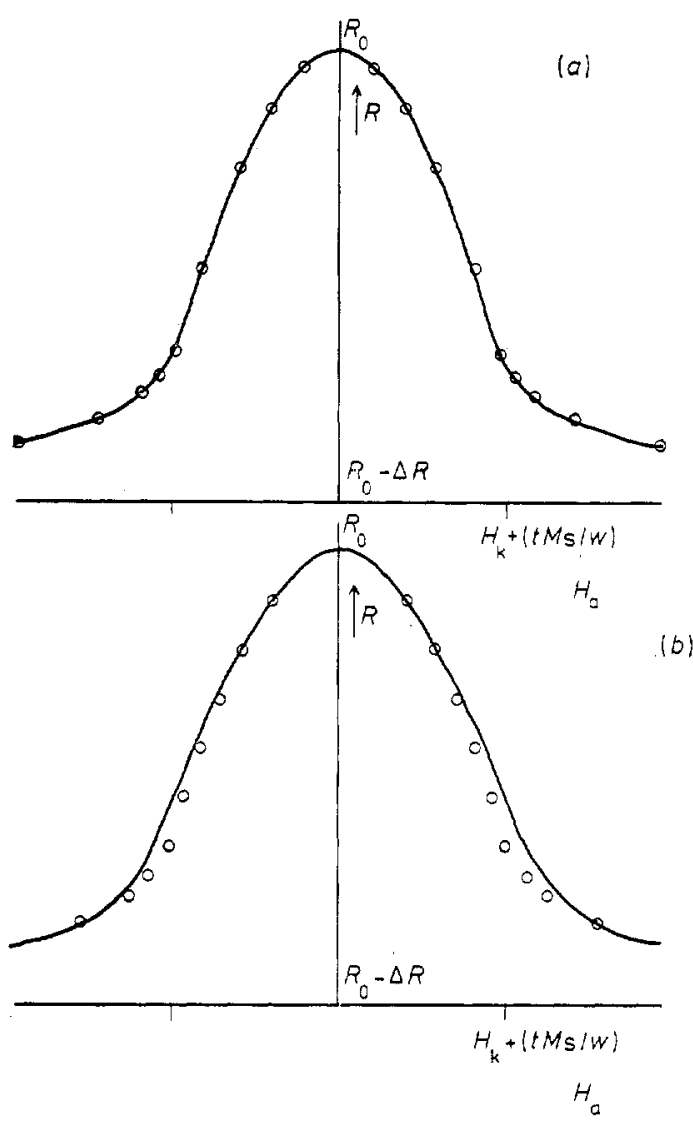

Figure 5 (a) Example of an experimental calibration curve (full curve) which fits to theory (open circles) over the entire range. (b) Example of an experimental calibration curve (full curve) which cannot be fitted to theory (open circles) over the entire range. In this case curves were fitted to overlap at least in the region of small fields but a slightly better fit can be obtained if the total field region is considered.

sensitivity. For a correct interpretation of the transducer response, the amplitude of the field component parallel to the strip axis has to be known. This is a drawback of the MRT, which must be used exclusively for the case of two-dimensional field patterns.

In the case of recording head fields however, this requirement is automatically fulfilled. We have investigated the influence of an externally applied field component along the strip axis during field measurements on a ferrite head. It appeared that such a component is effectively screened by the soft magnetic material of the head and no significant influence from such a field component has been found.

Another problem that may occasionally arise in measurements on soft magnetic structures is the magnetic image effect (the transducer may be imaged in the measured object). However, those effects are rather small, but in principle one can correct for them in the analysis of the results.

\section{The positioning system}

For the measurement of the perpendicular and tangential component of the magnetic field of a recording head for instance, the MRT is positioned above the head in the way of figure 6 . The head field is mapped out by moving the head for different, but fixed, values of the separation $h$ parallel to the lower surface of the substratum. The smallest separation that can be adjusted is determined by the size and the radius of curvature of the head surface and the occurrence of dust

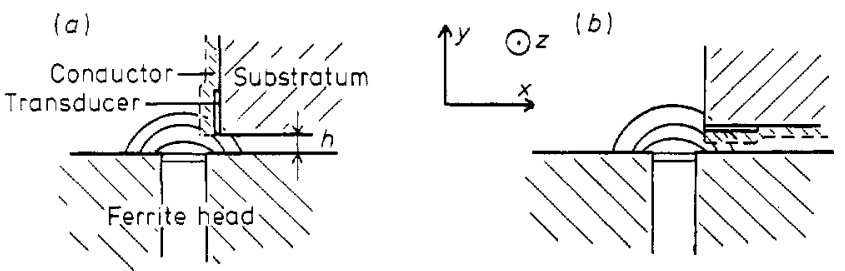

Figure 6 Positioning of the MRT substratum in the vicinity of the head gap (cross section) for measurement of (a) the perpendicular field component $H_{y}(x, y)$; and $(b)$ the tangential field component $H_{x}(x, y)$.

particles on the substratum and head surface. The smallest separation realised so far is less than $0.05 \mu \mathrm{m}$, when measuring the perpendicular field component (figure $6(a)$ ) of an audio head (radius of curvature $=0.01 \mathrm{~m}$ ) and a Winchester (tri-rail) disc slider head (area of the shortest pole piece $75 \times 75 \mu \mathrm{m}$ ). When measuring the tangential field component (figure $6(b)$ ), the smallest separation is determined by the thickness of the evaporated conductors on the substratum. Indeed in this position of the substratum careful manoeuvring of the MRT above the head surface is necessary in order to prevent damage to the transducer.

The essential parts of the positioner are:

\subsection{The mechanical system}

The construction for positioning the transducer and the head has to be very stable. Therefore, precautions have to be taken to mechanically and thermally isolate the system with respect to the laboratory environment. Yery precise and stable components are used for micropositioning the head with respect to the transducer. Essentially the system consists of a fixed and a movable part. One of the components (head or transducer) is mounted on the fixed part, while the other one is mounted on the movable part. For the movable part we have chosen a commercially available air bearing. The separation between the transducer substratum and the head surface can be adjusted with the help of the air pressure of the air bearing. It is an essential facility of the air bearing that its height can be adjusted over an interval of about $7 \mu \mathrm{m}$ in the $y$ direction in this way. A stepper motor with gear-case has been utilised for generation of the table motion (typical $1 \mu \mathrm{m} / \mathrm{s}$ in the $x$ direction).

\subsection{The system for determination of the table motion}

The displacement of the table in $x$ direction is measured by means of a Michelson-interferometer (Van Herk 1973). The accuracy of this method depends on the mechanical and thermal stability of the optical system parts and is typically $0.08 \mu \mathrm{m}$. However, alternative (electromechanical) methods of measuring the table motion accurately with a long range are commercially available by now.

\subsection{The system for determination of the separation $h$ between substratum and head surface}

The separation $h$ between head and substratum is measured with the help of optical interference using monochromatic light (Lin 1973). The accuracy with which the separation can be determined depends on the accuracy with which the position of an interference line is determined.

This is accomplished by the use of a video camera and display and a 'line selector' by which one or more video lines can be displayed on an oscilloscope screen (figure 7). By means of a vertical bar which is mixed into the video signal and which can 
(a)

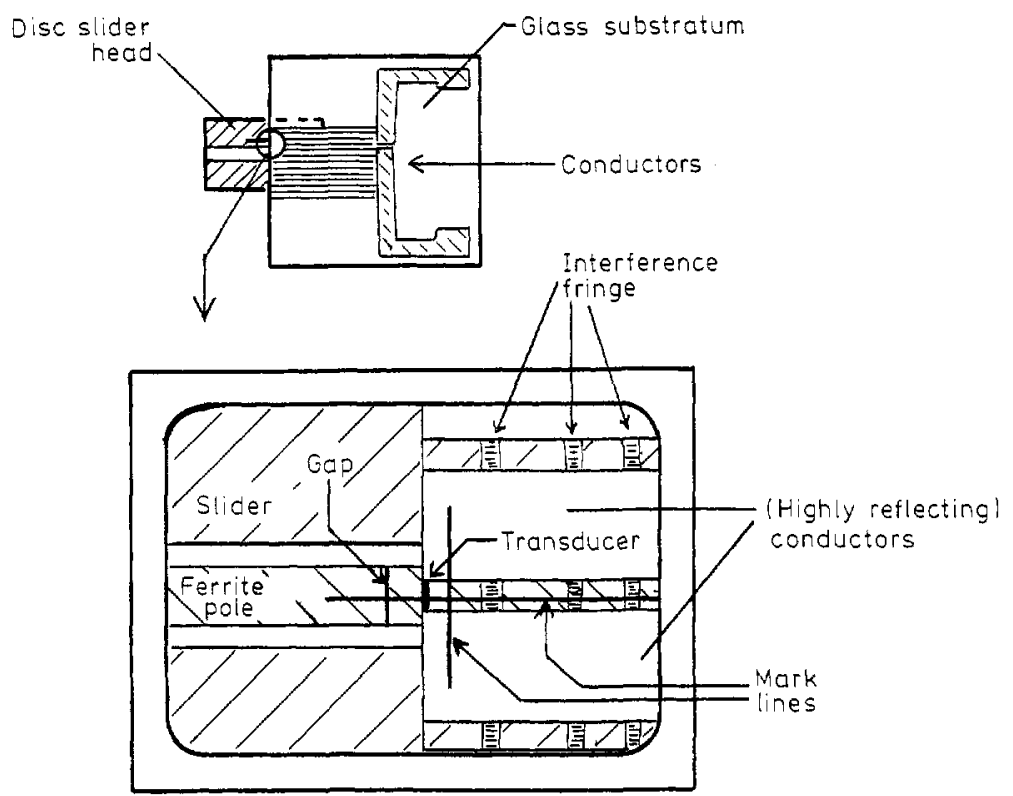

(c)

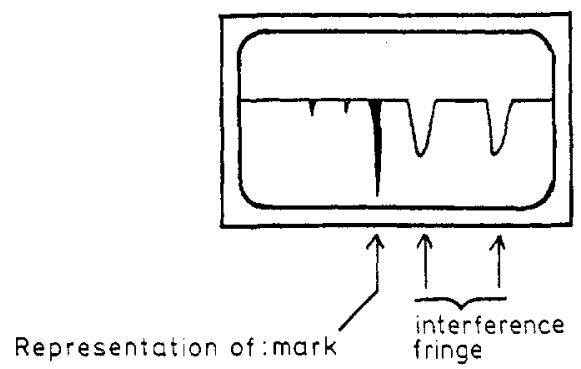

Figure 7 (a) Top view for measurement of the tangential field component of a 3330-type disc slider head; (b) monitor picture with mark lines mixed into the video image; (c) representation of the marked horizontal video line on an oscilloscope screen.

be moved over the screen like a cursor, an interference minimum or maximum can be selected on the oscilloscope screen, using the cursor as a parallax free pinnule. After positioning this bar another interference optimum can be positioned exactly on this mark by continuously changing the wavelength of the light using the procedure described by Lin (1973). In this way the value of $h$ can be determined with an accuracy of about $3 \%$. It is essential that the position of the interference lines relative to the transducer can be determined with sufficient accuracy. Use of a video recorder may be helpful to evaluate the measurements with respect to the separation afterwards.

Some problems with this optical system may arise when measuring tangential field components, because in this case the highly reflective evaporated conductors on the substratum mask the greater part of the interference pattern (figure 7). Therefore high demands are made upon the optical system concerning the perception of interference fringes through the spaces in the conductor pattern.

\section{Experiments on ferrite heads}

With the help of the equipment described above, we investigated the DC magnetic field of several commercial recording heads. Typical transducer responses in scanning the perpendicular and tangential field component are plotted in figure 8 as a function of the $x$ position for fixed separation $h$.

For correct results, the position of the MRT with respect to the head in $z$ direction has to be adjusted in such a way, that the side edges of the head do not influence the field component that is measured. Therefore the transducer length $L$ has to be smaller than the (track-)width of the head. The axis of the MRT-strip has to be aligned exactly parallel to the head gap,

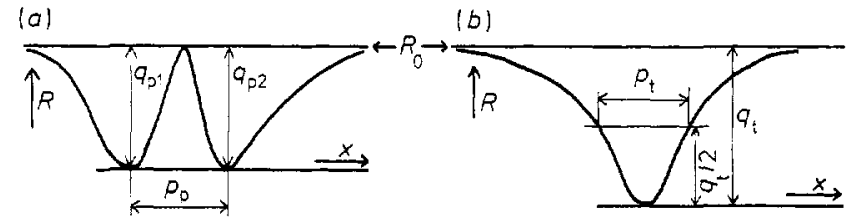

Figure 8 Typical transducer response as a function of the displacement for scanning: (a) the perpendicular field component; and $(b)$ tangential field component.

but in our experience this is hardly a problem. Azimuth errors are reduced to negligible values rather easily.

The occurrence of static magnetic stray fields in the region of detection must be reduced by demagnetising the ferrometallic system parts and the ferrite head beforehand. In our experiment the highly permeable ferrite head appeared to collect magnetic stray flux in such a way that a $y$ field component with a constant polarity could be identified for the whole head surface. However, this field can be compensated by means of a single coil surrounding the measurement system. The criterion for the correct adjustment of the compensation field is that the form of the response curve (see figure $8(a)$ ) remains unchanged after changing the polarity of the head current.

It is known that the $x$ and $y$ components of twodimensional field distributions are related to each other by Maxwell's laws. In the head field region, for instance, we have $\nabla . \boldsymbol{H}=0$ and $\nabla \times \boldsymbol{H}=\mathbf{0}$, and for $\boldsymbol{H}$ independent of $\boldsymbol{z}$ we find the relations:

$$
\frac{\partial H_{x}}{\partial x}=-\frac{\partial H_{y}}{\partial y} \quad \text { and } \quad \frac{\partial H_{x}}{\partial y}=\frac{\partial H_{y}}{\partial x} .
$$


Information about the magnetic field could be obtained from measurements on just one field component with the MRT. However, in view of the complicated relation between head field structure and MRT-response (see \$5), the accuracy of determining a field component is bound to be limited and analysis of the second field component can supply additional information more easily.

\section{Analysis of the results}

With the help of a transducer of width $w=2.5 \mu \mathrm{m}$, measurements have been made upon a series of four ferrite heads: a tri-rail (type 3348) disc slider head (with optical gap length $2 g=1.10 \mu \mathrm{m})$, an audio head $(2 g=1.85 \mu \mathrm{m})$ and two disc slider heads of the 3330 type $(2 g=2 \cdot 10$ and $2 \cdot 80 \mu \mathrm{m})$. We measured both field components of the audio and type 3330 disc slider heads and only the perpendicular field component of the tri-rail head. In order to prevent transducer saturation we have used rather small values of the (DC) drive currents through the head coil (about $10 \%$ of the operating values), but transducers can be prepared for measurement of larger fields.

In the analysis of the results we have not tried to derive field distributions from the observed response curves directly. Instead we have computed theoretical response curves with the help of a program simulating the MRT (Fluitman 1978) in a head field that can be chosen at will as an input to the simulation program. The first estimate has been the field distribution of an ideal infinite pole tip head (Westmijze 1953) with a gap length as it is observed optically.

The direct computation of headfields from response curves is not adequate at the moment. The straightforward computation of the MRT response from a given field component already takes $3 \mathrm{~min} / 40$ kbyte (about $100 \mathrm{MRT}$ positions) on a DEC-10 computer and one can imagine the huge computing capacity needed for a program thai automatically searches for a good fitting field input.

The response curves as they have been computed show the same structure as the experimental ones, but they generally differ in width (to be defined hereafter). The procedure we have followed in our analysis has been to change the input field of the computer simulation by introducing an effective gap length (the gap length needed to fit theory to experiment) or an effective head/transducer separation. Both methods work and lead to reasonable fits. In this procedure we have defined curve parameters which are adequate in testing the quality of

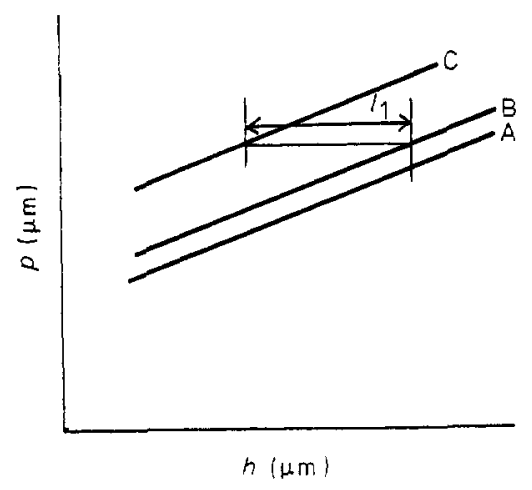

Figure 9 Adopting the model with dead layers $l_{1}$ and $l_{2}$ (figure 10), it is possible to fit the computed and measured $p$-values. A, computed values for gap length $2 g ; \mathrm{B}$, computed values for gap length $2 g+2 l_{2} ; \mathrm{C}$, measured values (optical gap length $2 g$ ). a fit. Agreement in the values of these parameters proved to be a guarantee for a good fit of the entire response curves. They are defined in figure 8 . The parameters $p_{\mathrm{p}}$ and $p_{\mathrm{t}}$ (indicating the 'width' of the curves) are much less sensitive to errors than the amplitude parameters $q_{\mathrm{p}}$ and $q_{\mathrm{t}}$ (even in the case of $p_{\mathrm{t}}$ whose determination depends on $q_{\mathrm{t}}$ ).

The $p_{p}$ and $p_{t}$ values of the computed and measured response curves have been plotted as a function of the separation $h$ between substratum and head surface. All of the plots have the characteristics of figure 9 . We have tried to associate these experimental phenomena with a 'dead layer' model (Fluitman and Groenland 1979) as suggested in figure 10.

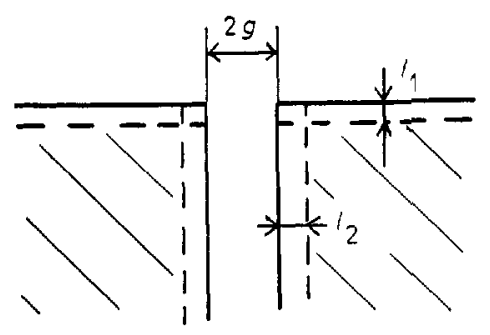

Figure 10 Cross section of a recording head with dead layer of thickness $l_{1}$ on the surface of the ferrite body and thickness $l_{2}$ in the gap.

The assumption of a dead layer on the head surface (thickness $l_{1}$ ) can bring the theoretical results into agreement with the experimental results because an inactive surface layer implies a shift along the $h$-axis of the diagram. Dead layers inside the gap (thickness $l_{2}$ ) may also bring the results into agreement. In that case one has to choose a value of $l_{2}$, such that results of a computation based on an effective gap length $2 g+2 l_{2}$ fit to the experimental curve. This can be done but it must be said that the results are less sensitive to $l_{2}$ than to $l_{1}$.

In general one must assume all layers to be present and look for values of $l_{1}$ and $l_{2}$ as is suggested in figure 9. However, there are infinitely many pairs of $l_{1}, l_{2}$ values that lead to a fit. We tried to find a couple of $l_{1}, l_{2}$ values that lead to a fit in both the perpendicular and the tangential case. The procedure is demonstrated in figure 11 , where we plotted the relation between the determined $l_{1}, l_{2}$ values.

For the audio head we found values for the dead layer thicknesses $\left(l_{1}=0.5 \mu \mathrm{m}, \quad l_{2}=0.6 \mu \mathrm{m}\right)$ which obeyed the proposed dead layer model. However, in contradiction to this model we found the $p$-values to depend on the head current for this recording head. For the 3330 heads the described procedure leads to very large values of $l_{2}$ while $l_{1}$ is very close to zero. Although such an outcome may reflect the real situation, we consider it as rather improbable and suggest that the model and/or the method of analysis needs refinement. From figure 11 one can see what values of dead layer thicknesses are to be considered in the first instance.

A refinement of the model can be worked out in several ways, for instance in a more gradual or curved layer boundary, in the acceptance of specific magnetic behaviour (e.g. anisotropy) of the layer (which is not really 'dead' in that case), and so on. It is known from literature that surface machining of $\mathrm{Mn}-\mathrm{Zn}$ ferrite single crystals, for instance, may give rise to a variety of surface phenomena (Kinoshita et al 1976) and we have strong experimental evidence that the magnetic layers may have a peculiar magnetic structure indeed.

Measurements of the perpendicular field component of the tri-rail and the audio-head have been made at a very small distance from the head surface $(h \sim 0.05 \mu \mathrm{m})$. For both heads 


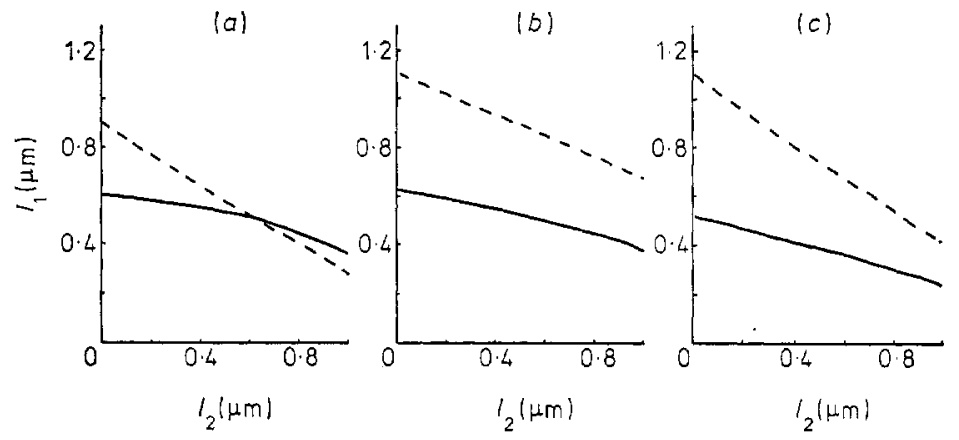

Figure 11 Relation between the values of the "dead layers' $l_{1}$ and $l_{2}$ to fit the computed $p$ to $h$ curves to the experimental ones (- perpendicular, - - tangential field component). (a) Audio head $(2 g=1.85 \mu \mathrm{m}) ;(b)$ type 3330 disc slider head $(2 g=2 \cdot 10 \mu \mathrm{m})$; (c) same type $(2 g=2 \cdot 80 \mu \mathrm{m})$. a remarkable transducer response could be observed (figure 12).

The transducer detected static magnetic field concentrations which in first order proved to be independent of amplitude and direction of the head current. The effect disappeared for increasing value of the separation $h$. We guess that magnetised spots are situated in the ferrite surface, with such a size and

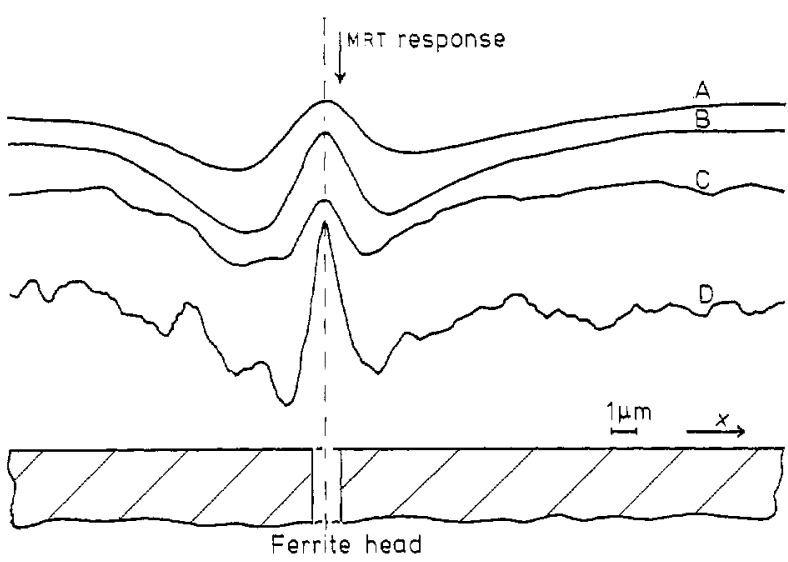

Figure 12 MRT response on the perpendicular field component of a tri-rail head $(2 g=1 \cdot 10 \mu \mathrm{m})$ for: A, separation $h=2.0 \mu \mathrm{m}$, head current $=12 \mathrm{~mA}$; $\mathrm{B}, h=1.25 \mu \mathrm{m}$, head current $=12 \mathrm{~mA} ; \mathrm{C}, h=0.5 \mu \mathrm{m}$, head current $=7 \mathrm{~mA} ; \mathrm{D}, h \simeq 0.05 \mu \mathrm{m}$, head current $=7 \mathrm{~mA}$.

distribution that their effect is detectable in the transducer response in spite of the averaging behaviour of the MRT over its length $L$. The so-called dead layer seems to be built up out of a distribution of hard magnetic particles. (In the analysis concluding about dead layers $l_{1}$ and $l_{2}$, we have only made use of the smooth curves obtained for values of the separation $h>1 \mu \mathrm{m}$.)

It is beyond the aim of this work to develop a more detailed model to explain the measured responses exactly and one can even wonder if such a thing is possible at all for a set of different heads. However, we can conclude that heads which are commercially available may have degraded layers with thicknesses of several tenths of a $\mu \mathrm{m}$.

\section{Conclusions}

We have developed and described a measurement system capable of analysing two-dimensional field patterns beyond the $\mu \mathrm{m}$ level even with a transducer that is relatively large. It has been shown that it is possible to make measurements of recording head fields and the description of the system may inspire application in other studies.

With respect to recording heads the system can give information about the head field structure and implicitly about (possible) asymmetry, remanence, efficiency and head saturation phenomena. It has been shown that the microscopic surface structure of heads can be detected and it is concluded that commercially available heads may have degraded surface layers of considerable thickness.

\section{Acknowledgments}

Many co-workers and students in our laboratory have contributed to this project that has run for several years. We would like to mention by name A J F Hollink and P D Ekkelkamp (technical support). The measurement frame has been constructed by A Bakker and the transducers produced by B Hurenkamp and $\mathrm{H}$ W Krabbe.

\section{References}

Fluitman J H J 1978 Recording head field measurement with a magneto-resistive transducer

\section{IEEE Trans. Magn. 14433}

Fluitman J H J and Groenland J P J 1979 Investigation of the structure of recording head fields

IEEE Trans. Magn. 151634

Fluitman J H J and Krabbe H W 1972 Miniature sensor for two-dimensional magnetic field distributions

J. Phys. E: Sci. Instrum. 5963

van Herk A 1973 Use of an $X Y$ oscilloscope in interferometric measurements of small displacements J. Phys. E: Sci. Instrum. 6612

Kinoshita M, Murayama T, Hoshina N, Kobayashi A, Shimizu R and Ikuta T 1976 The surface damaged layer study of $\mathrm{Mn}-\mathrm{Zn}$ single crystal ferrites using magnetic domain observation Annals CIRP 25449

Lin C 1973 Techniques for the measurement of air-bearing separation - a review

IEEE Trans. Magn. 9673

Lustig C D, Baird A W, Chaurette W F, Minden H, Maloney W T and Kurtzig A J 1979 High-resolution magnetic field measurement system for recording heads and disks

Rev. Sci. Instrum. $\mathbf{5 0} 321$

West F G 1961 Magnetoresistive measurements on domain rotation and wall development in Ni-Fe alloy films J. Appl. Phys. 32 290S

Westmijze W K 1953 Studies on magnetic recording Philips Res. Rep. 8148 